\title{
Luteolin inhibits angiogenesis by blocking Gas6/Axl signaling pathway
}

\author{
XIAOBO LI $^{1,2^{*}}$, MINFENG CHEN ${ }^{1,2^{*}}$, XUEPING LEI $^{1,2}$, MAOHUA HUANG $^{1,2}$, \\ WENCAI YE ${ }^{1,2}$, RIJIA ZHANG ${ }^{3}$ and DONGMEI ZHANG ${ }^{1,2}$ \\ ${ }^{1}$ College of Pharmacy, Jinan University; ${ }^{2}$ Guangdong Province Key Laboratory of Pharmacodynamic \\ Constituents of Traditional Chinese Medicine and New Drugs Research, Jinan University; \\ ${ }^{3}$ The First Affiliated Hospital of Jinan University, Guangzhou, Guangdong 510632, P.R. China
}

Received April 15, 2017; Accepted May 26, 2017

DOI: $10.3892 /$ ijo.2017.4041

\begin{abstract}
Growth arrest-specific protein 6 (Gas6) induces the activation of $A x l$ receptor tyrosine kinase (Axl), which plays an important role in angiogenic processes, including proliferation, migration, invasion, tube formation and pericyte recruitment of endothelial cells. The inhibition of Gas6/Axl pathway has been demonstrated to be an effective anti-angiogenic therapy. Luteolin, which is a natural active flavonoid, has been reported to possess anti-angiogenic effects. However, the underlying mechanism of luteolin in anti-angiogenesis is not fully understood. Herein, we
\end{abstract}

Correspondence to: Dr Dongmei Zhang, College of Pharmacy, Jinan University, 601 Huangpu Road West, Guangzhou, Guangdong 510632, P.R. China

E-mail:dmzhang701@foxmail.com

Dr Rijia Zhang, The First Affiliated Hospital of Jinan University, 613 Huangpu Road West, Guangzhou, Guangdong 510632,P.R. China E-mail: jerry_joan@163.com

*Contributed equally

Abbreviations: HMEC-1s, human microvascular endothelial cells; HBVPs, human brain vascular pericytes; ECs, endothelial cells; Gas6, growth arrest-specific protein 6; Axl, Axl receptor tyrosine kinase; VEGF, vascular endothelial growth factor; EGF, epidermal growth factor; FGF, fibroblast growth factor; PDGF, plateletderived growth factor; Ang1, angiopoietin 1; Ang2, angiopoietin 2; VEGFR2, vascular endothelial growth factor receptor 2; PI3K, phosphatidylinositol 3-kinase; Akt, protein kinase B; mTOR, mammalian target of rapamycin; RTK, receptor tyrosine kinases; MMP-2, matrix metalloproteinase-2; MMP-9, matrix metalloproteinase-9; HSP90, heat shock protein 90; PM, pericyte medium; MEK, mitogen-activated protein kinase; ERK, extracellular signal-regulated kinase; NF- $\kappa \mathrm{B}$, nuclear factor $\kappa \mathrm{B} ; \mathrm{JAK}$, janus kinase; STAT, signal transducer and activator of transcription; CAM, chick chorioallantoic membrane; MTT, 3-(4, 5-dimethylthiazol-2-yl)-2, 5-diphenyltetrazolium bromide

Key words: luteolin, angiogenesis, Gas6, Axl report that luteolin significantly inhibited the Gas6-induced proliferation, migration, invasion and tube formation of human microvascular endothelial cells (HMEC-1s) in vitro, and suppressed the Gas6-induced recruitment of human brain vascular pericytes (HBVPs) to the endothelial tubes. Luteolin also suppressed Gas6-induced microvessel sprouting in aortic ring assay and neovascularization in chick chorioallantoic membrane assay. The anti-angiogenic effect of luteolin may be associated with the inhibition of the Gas6/Axl pathway and its downstream phosphatidylinositol 3-kinase (PI3K)/protein kinase B (Akt)/mammalian target of rapamycin (mTOR) signaling pathways. Taken together, the present study provides new evidence regarding an antiangiogenic mechanism of luteolin, and supports the notion that the dietary intake of luteolin contributes to the treatment of pathological angiogenesis.

\section{Introduction}

Angiogenesis occurs when new vessels are generated from pre-existing vessels, this involves a complicated process, including vascular destabilization, extracellular matrix degradation, endothelial cell proliferation, migration, invasion, tube formation and the recruitment of pericytes. Multiple pro-angiogenic and anti-angiogenic factors, such as vascular endothelial growth factor (VEGF), epidermal growth factor (EGF), fibroblast growth factor (FGF), platelet-derived growth factor (PDGF), angiopoietin 1 (Ang1) and angiopoietin 2 (Ang2), are involved in the regulation of angiogenesis $(1,2)$. Physiological angiogenesis results from a fine-tuning balance between these pro-angiogenic and anti-angiogenic factors and establishes a well-organized vessel network to provide oxygen and nutrients to tissues. When the balance is disturbed, vessel growth is deregulated, resulting in physiological immature vessels, which may lead to pathological angiogenesis, including cancer, psoriasis, blindness, and arthritis $(2,3)$. To inhibit pathological angiogenesis, several inhibitors and monoclonal antibodies targeting pro-angiogenic factors have been developed and applied in clinic. However, there are limitations to these anti-angiogenic agents, such as serious side effects and drug resistance, which attenuate their efficacy and hamper drug development $(4,5)$. 
Growth arrest-specific protein 6 (Gas6), which is a member of the vitamin K-dependent protein family, is an important pro-angiogenic factor. Gas6 has different affinities for TyroAxl-Mer (TAM) receptor tyrosine kinases (RTKs) and shows selectively high affinities for Axl receptor tyrosine kinase (Axl) (6). The Gas/Axl axis is expressed in endothelial cells (ECs), pericytes, and smooth muscle cells (7-9), and has been demonstrated to participate in multiple angiogenic processes including survival, proliferation, migration, invasion and aggregation of ECs as well as pericyte adhesion by regulating its downstream signaling pathways, including the phosphatidylinositol 3-kinase (PI3K)/protein kinase B (Akt)/mammalian target of rapamycin (mTOR) pathway, the mitogen-activated protein kinase (MEK)/extracellular signal-regulated kinase (ERK) pathway, the nuclear factor- $\kappa \mathrm{B}(\mathrm{NF}-\kappa \mathrm{B})$ pathway and the janus kinase (JAK)/signal transducer and activator of transcription (STAT) pathway (10-13). Previously, many preclinical studies have shown that the inhibition of the Gas6/ Axl pathway may contribute to reduction in angiogenesis (9). R428, which is a selective inhibitor of the Axl kinase, has been demonstrated to block Axl-dependent events, such as Akt phosphorylation, breast cancer cell invasion, and proinflammatory cytokine production (14). Desacetylvinblastine monohydrazide, which is a derivative of vinblastine, has also been reported to suppress angiogenesis in vitro and in vivo by suppressing the Gas6/Axl signaling pathway (15). In addition, the inhibition of Gas6/Axl pathway enhances the effects of multiple anti-angiogenic therapies in tumors (16). Altogether, these findings suggest that the Gas/Axl pathway is a therapeutic target for pathological angiogenesis.

Luteolin (3', 4', 5, 7-tetrahydroxy flavone) is widely distributed in vegetables and fruits and serves as a common dietary additive (17). Preclinical studies have demonstrated that luteolin has multiple pharmacological activities, such as antiinflammation, anti-tumor, and anti-microbial activities (18). Moreover, luteolin dietary additive has been considered favorable in many pathological angiogenesis therapies (19). Previously, luteolin has been shown to inhibit tumor angiogenesis, including human lung cancer, breast cancer, and prostate tumor, however, the mechanism underlying this inhibition remains to be elucidated (20). In the present study, we reveal that luteolin suppresses Gas6-induced angiogenesis in vitro, ex vivo and in vivo by downregulating the Gas6/Axl signaling pathway. Our results show that luteolin inhibits Gas6-mediated proliferation, motility and tube formation of human microvascular endothelial cells (HMEC-1s), which are required for neovascularization, and Gas6-induced recruitment of pericytes during vessel maturation. In addition, using an aortic ring assay and a chick chorioallantoic membrane (CAM) assay, we demonstrate that luteolin suppresses Gas6induced vascular sprouting and neovascularization.

\section{Materials and methods}

Materials. Luteolin ( $\geq 98 \%$ pure) was purchased from Sigma-Aldrich (St. Louis, MO, USA), dissolved in DMSO and stored at $-20^{\circ} \mathrm{C}$. Fetal bovine serum (FBS) was obtained from Life Technology (Carlsbad, CA, USA). Pentobarbital sodium was purchased from Merck (Darmstadt, Germany). Matrigel was purchased from BD Biosciences (Franklin Lakes, NJ,
USA). The antibodies against Axl, PI3K, p-PI3K ${ }^{\text {Tyr458 }}$, Akt, p-Akt $^{\text {Thr308 }}$, mTOR, p-mTOR ${ }^{\text {Ser2448 }}$, p70S6k, p-p70S6k ${ }^{\text {Thr389 }}$, heat shock protein 90 (HSP90), matrix metalloproteinase 2(MMP-2), matrix metalloproteinase 9 (MMP-9), and donkey anti-Rabbit IgG were purchased from Cell Signaling Technology (Danvers, MA, USA). The p-Axl ${ }^{Y 779}$ antibody and recombinant human Gas6 were obtained from R\&D Systems (Minneapolis, MN, USA). The pericyte medium (PM) was obtained from ScienCell Research Laboratories (San Diego, CA, USA). The fertilized chicken eggs were purchased from South China Agricultural University (Guangzhou, China). PKH 26, PKH 67 and other reagents were purchased from Sigma-Aldrich.

Cell lines and cell culture. Human microvascular endothelial cells (HMEC-1s) were purchased from American Type Culture Collection (ATCC, Manassas, VA, USA) and cultured in RPMI-1640 medium (Gibco, Invitrogen Corp., Carlsbad, CA, USA) supplemented with $10 \%$ FBS. HBVPs were obtained from ScienCell Research Laboratories and cultured in PM. All cells were cultured at $37^{\circ} \mathrm{C}$ under humidified atmosphere containing $5 \% \mathrm{CO}_{2}$.

Animals. Adult male Sprague Dawley rats (weighing 180-220 g) were obtained from the Guangdong Medical Experimental Animal Center (Guangzhou, China). The animals were maintained in a specific pathogen-free room with free access to water and standard laboratory chow. All animal experiments were approved by the laboratory animal ethics committee of Jinan University (Guangzhou, China).

Cell viability assay. The effect of luteolin on the viability of HMEC-1s was assessed using the 3-(4, 5-dimethylthiazol2-yl)-2, 5-diphenyltetrazolium bromide (MTT) assay. HMEC-1s were seeded in 96-well plates at $1 \times 10^{4}$ cells per well and cultured for $24 \mathrm{~h}$. Then, the cells were treated with various concentrations of luteolin. After incubating for 24 and $48 \mathrm{~h}$, the cell viability was determined using the MTT assay. The Gas6-induced HMEC-1s proliferation was measured by the MTT assay as previously described (21). HMEC-1s were seeded in 96-well plates at $1 \times 10^{4}$ cells per well and cultured for $24 \mathrm{~h}$. Adherent cells were starved with serum-free RPMI-1640 medium for $6 \mathrm{~h}$, then, the cells were treated with various concentrations of luteolin, and Gas6 (100 ng/ml) was simultaneously added. After incubating for 24 and $48 \mathrm{~h}$, the cell viability was determined using the MTT assay.

Wound-healing migration assay. HMEC-1s were grown to $100 \%$ confluence in 6 -well plates. The cells were starved with serum-free RPMI-1640 medium for $6 \mathrm{~h}$, and then scratched with pipette tips. The cells were cultured with $1 \%$ FBS RPMI-1640 medium containing various dilutions of luteolin in the presence or absence of Gas6 $(100 \mathrm{ng} / \mathrm{ml})$ for $8 \mathrm{~h}$. Images of the cells were taken 0 and $8 \mathrm{~h}$ after wounding.

Transwell migration and Matrigel invasion assay. The in vitro migration and invasion were assessed using a Transwell assay with or without Matrigel as previously described (22). Briefly, $2 \times 10^{4}$ HMEC-1s were suspended in $100 \mu \mathrm{l}$ of the serum-free RPMI-1640 medium with or without various concentrations of luteolin (10 and $20 \mu \mathrm{M})$ added to the upper chamber insert, the 

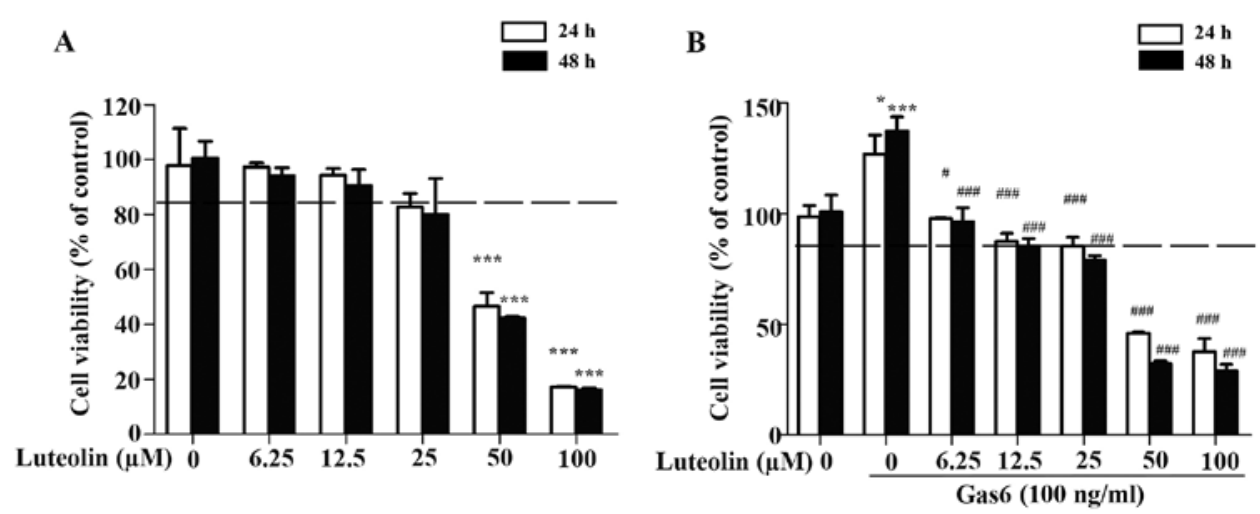

Figure 1. Luteolin inhibits proliferation of HMEC-1s in a dose-dependent manner. (A) An MTT assay revealed that luteolin suppressed the proliferation of HMEC-1s in a dose-dependent manner. Adherent HMEC-1s were treated with different concentrations of luteolin. Cell viability was detected by the MTT assay. (B) Luteolin suppressed the Gas6-induced proliferation of HMEC-1s. HMEC-1s were starved for $6 \mathrm{~h}$ and then treated with different concentrations of luteolin with or without Gas6 $(100 \mathrm{ng} / \mathrm{ml})$ for 24 and $48 \mathrm{~h}$. Then, the cell viability was detected by the MTT assay. The data are presented as the mean \pm SEM. ${ }^{*} \mathrm{P}<0.05,{ }^{* * *} \mathrm{P}<0.001$ compared with the control group; ${ }^{*} \mathrm{P}<0.05,{ }^{\# \#} \mathrm{P}<0.001$ compared with the Gas6-treated group.

bottom chamber was filled with $600 \mu 1$ of fresh RPMI-1640 medium supplemented with or without Gas6 (100 $\mathrm{ng} / \mathrm{ml})$. After 24-h incubation, the upper chamber was fixed with $4 \%$ paraformaldehyde for $30 \mathrm{~min}$ and the cells were stained with $0.1 \%$ crystal violet. The non-migrated HMEC-1s that remained on the inner side of the upper chamber were removed with a cotton swab. The cells on the lower surface were photographed under an Olympus IX70 inverted microscope. For the cell invasion assay, the upper chamber was pre-coated with $35 \mu \mathrm{l}$ of diluted Matrigel (Matrigel was diluted to a ratio of 3:1 with PBS) for $1 \mathrm{~h}$ at $37^{\circ} \mathrm{C}$, and the assay was performed according to the procedures described above. The migrated and invasive cells were quantified using Image-Pro Plus 6.0.

Tube formation assay. The HMEC-1s were seeded in Matrigel-coated 96-well plates at a density of $2.5 \times 10^{4}$ cells per well with or without the presence of the indicated concentrations of luteolin $(10$ and $20 \mu \mathrm{M})$, and Gas6 $(100 \mathrm{ng} / \mathrm{ml})$ was simultaneously added. After $8 \mathrm{~h}$, capillary-like tubes were observed and photographed under an Olympus IX70 inverted microscope, and the number of tubes was calculated by Image-Pro Plus 6.0.

Aortic ring assay. The dorsal aortas were isolated from the Sprague-Dawley rats as previously described (15). Then the dorsal aortas were cut into 1- to 1.5-mm-long rings. Next, the rinsed aortic rings were placed in Matrigel-coated 96-well plates and sealed with an overlay of $60 \mu 1$ of Matrigel. After 2-h incubation, fresh RPMI-1640 medium containing Gas6 (300 $\mathrm{ng} / \mathrm{ml}$ ) was added, and the rings were incubated for $48 \mathrm{~h}$. Then, the aortic rings were treated with luteolin $(10 \mu \mathrm{M}$ or $20 \mu \mathrm{M}$ ) for 7 days. The microvessels were photographed under an Olympus IX70 inverted microscope, and the microvessel structures that extend outward from the aortic ring were quantified using Image-Pro Plus 6.0.

Three-dimensional co-cultures of HMEC-1s and HBVPs. The three-dimensional (3D) co-culture of the HMEC-1s and HBVPs was performed as previously described with slight modifications (23). Briefly, the HMEC-1s were labeled with PKH 26 $(\lambda \mathrm{ex}=551 \mathrm{~nm}, \lambda \mathrm{em}=567 \mathrm{~nm})$ following the manufacturer's instructions. Next, $2 \times 10^{4}$ HMEC-1s were seeded into Matrigelcoated 96-well plates and incubated for $2 \mathrm{~h}$ to allow the capillary network to form. Then, $2 \times 10^{4}$ HBVPs, which were pre-treated with luteolin for $4 \mathrm{~h}$ and labeled with PKH $67(\lambda \mathrm{ex}=490 \mathrm{~nm}$, $\lambda \mathrm{em}=502 \mathrm{~nm}$ ), were added to the capillary network and incubated for another $10 \mathrm{~h}$ to allow the HBVPs to adhere to the capillary network and form complicated and solid tubes. The capillary networks were observed and captured under an Olympus IX70 inverted microscope at 0,2 and $10 \mathrm{~h}$ after the addition of HBVPs.

CAM assay. The CAM assay was performed as previously described with slight modifications (24). Fertilized chicken eggs were incubated at $37.8^{\circ} \mathrm{C}$ in a humidified incubator containing 60-65\% humidity for 5 days (blunt end up). A small window approximately $1 \mathrm{x} 1 \mathrm{~cm}$, was carefully drilled in the eggshell on the gas chamber side to create an artificial gas chamber. Then, the shell and shell membrane were removed to expose the CAM. Next, a piece of filter membrane containing Gas6 $(300 \mathrm{ng} / \mathrm{ml})$ and the indicated concentrations of luteolin (10 and $20 \mu \mathrm{M}$ ) dissolved in $20 \mu \mathrm{l} \mathrm{PBS}$, was placed in the center of the CAM. The window was sealed with transparent tape and the CAM was incubated for another $48 \mathrm{~h}$. The microvessels in the CAM were observed under an Olympus SZX18 dissecting microscope, and the microvessels were quantified with Image-Pro Plus 6.0.

Western blot analysis. HMEC-1s were starved for $6 \mathrm{~h}$ in serum-free RPMI-1640 medium and HBVPs were treated with serum-free PM in the same way, then the cells were treated with luteolin $(10$ and $20 \mu \mathrm{M})$ for $4 \mathrm{~h}$. After luteolin was removed, cells were stimulated with Gas6 $(100 \mathrm{ng} / \mathrm{ml})$ for another $1 \mathrm{~h}$. Then, the cells were harvested and lysed with RIPA buffer at $4^{\circ} \mathrm{C}$. Proteins were analyzed by electrophoresis on $10 \%$ SDS-polyacrylamide gels and then detected by western blot as previously described (25). Protein gray values were measured with ImageJ software (NIH, Bethesda, MD, USA).

Statistical analysis. All experiments were performed in triplicate. The data are expressed as the mean \pm SEM, and the statistical analyses were performed with GraphPad Prism 5.0 
A
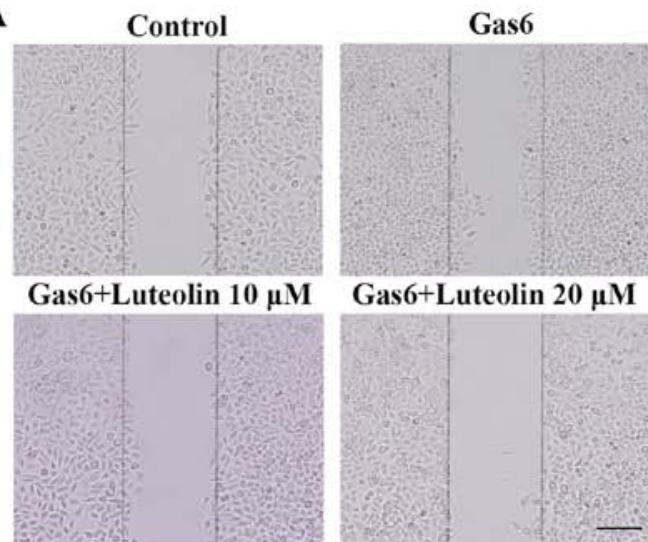

Gas6+Luteolin $20 \mu \mathrm{M}$

B

Control
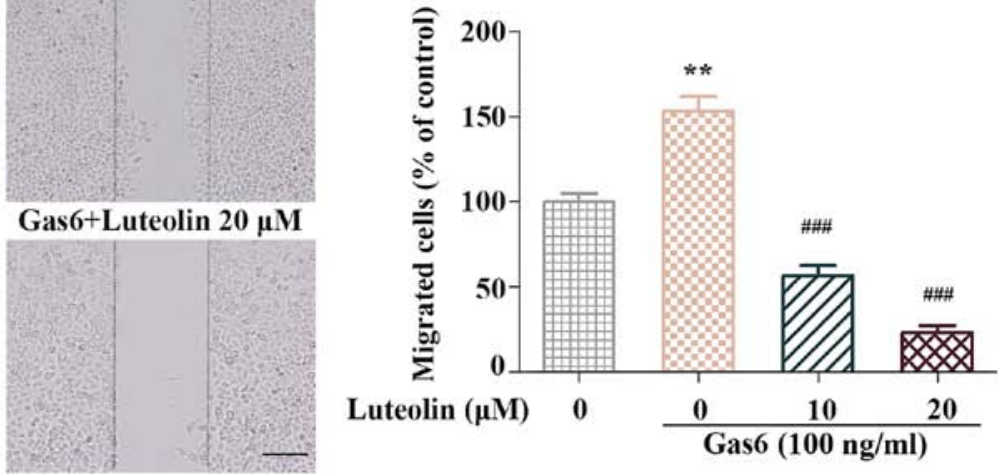

Luteolin $(\mu \mathrm{M}) \quad 0$
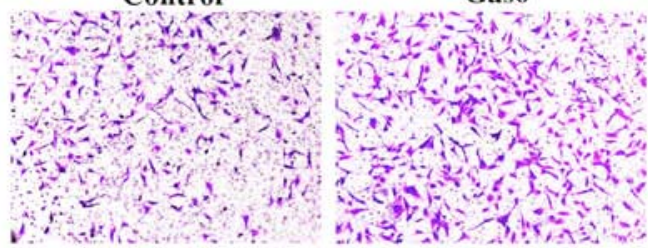

Gas6+Luteolin $10 \mu \mathrm{M}$
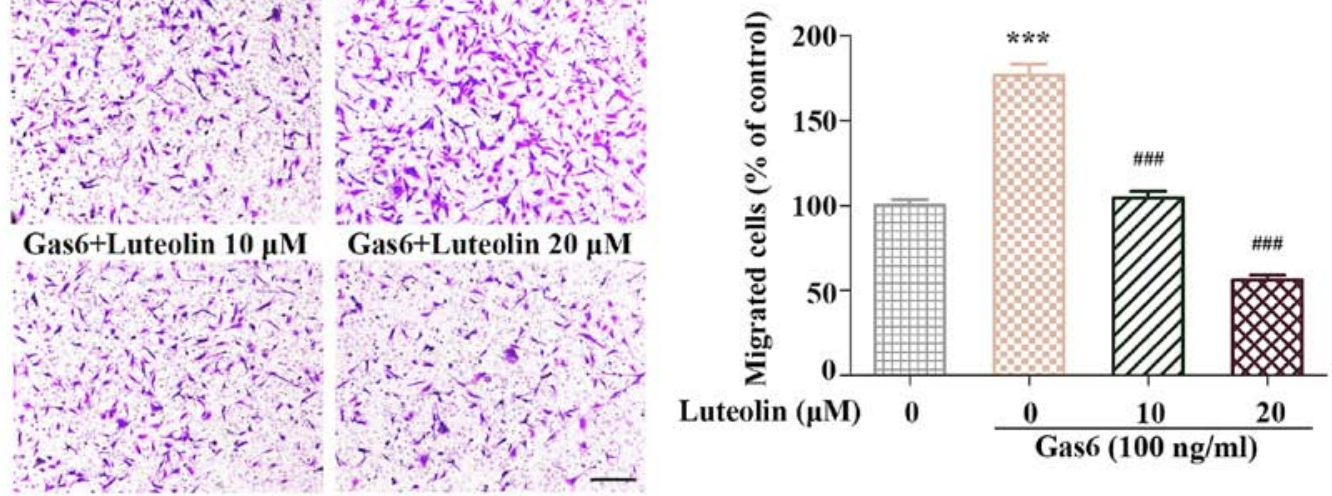

C

Control

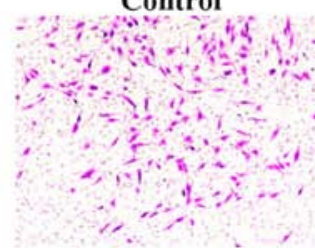

Gas6+Luteolin $10 \mu \mathrm{M}$

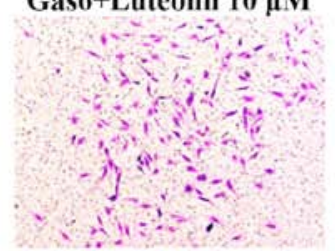

Gas6

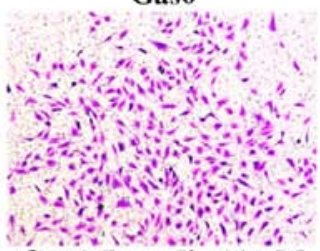

Gas $6+$ Luteolin $20 \mu \mathrm{M}$

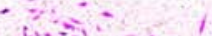

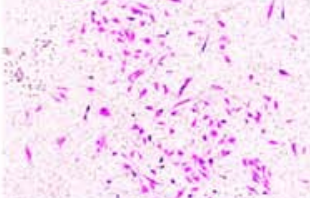

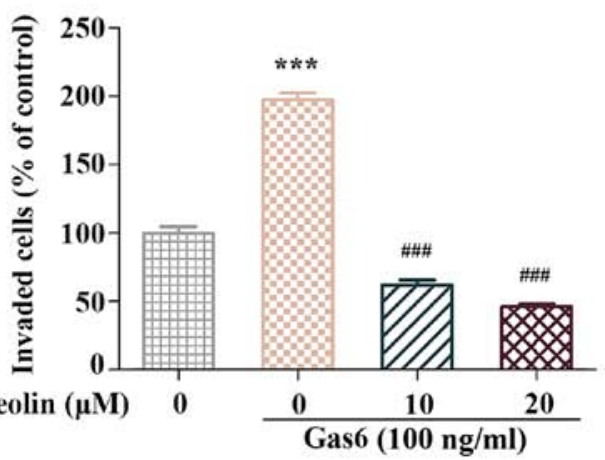

Figure 2. Luteolin suppresses the Gas6-mediated angiogenesis in vitro. (A) Luteolin inhibited the Gas6-induced migration of HMEC-1s in the wound-healing assay. Scratched HMEC-1s were treated with luteolin in the presence or absence of Gas6 (100 ng/ml) for $8 \mathrm{~h}$. The images were taken under an Olympus IX70 inverted microscope at 0 and $8 \mathrm{~h}$, and the migrated cells were quantified using Image-Pro Plus 6.0. (B) Luteolin suppressed the Gas6-induced migration of HMEC-1s in the Transwell assay. HMEC-1s $\left(2 \times 10^{4}\right)$ were suspended with serum-free RPMI-1640 with or without luteolin added to the upper chamber of Transwell and the lower chamber were filled with $0.6 \mathrm{ml}$ fresh RPMI-1640 in the presence or absence of Gas6 (100 ng/ml). After $24 \mathrm{~h}$, the upper chamber was fixed with $4 \%$ paraformaldehyde for $30 \mathrm{~min}$, and the cells were stained with crystal violet. The cells that did not migrate to the inner side were removed with a cotton swab. The migrated cells were photographed under an Olympus IX70 inverted microscope and quantified using Image-Pro Plus 6.0. (C) Luteolin significantly inhibited the Gas6-induced HMEC-1s invasion. The upper chamber was coated with Matrigel and the following procedures were performed according to the Transwell migration assay. Representative images are shown, scale bar, $50 \mu \mathrm{M}$. The data are presented as mean $\pm \mathrm{SEM}$. ${ }^{* *} \mathrm{P}<0.01,{ }^{* * *} \mathrm{P}<0.001$ compared with the control group; ${ }^{\# \#} \mathrm{P}<0.001$ compared with the Gas6-treated group.

(GraphPad Software, La Jolla, CA, USA). Significant differences were evaluated with one-way ANOVA followed by Tukey's test. $\mathrm{P}<0.05$ was considered statistically significant.

\section{Results}

Luteolin inhibits the Gas6-induced proliferation of HMEC-1s. First, the non-toxic concentration of luteolin in HMEC-1s was determined by the MTT assay. Our results showed that luteolin had a negligible effect on the proliferation of HMEC-1s at a dose range of 10 to $20 \mu \mathrm{M}$ (Fig. 1A). Therefore, the concentrations of 10 and $20 \mu \mathrm{M}$ were identified as non-toxic doses and used in the subsequent in vitro, ex vivo and in vivo experiments. Then, we examined the effect of luteolin on the Gas6-induced proliferation of HMEC-1s. We found that Gas6 significantly promoted the proliferation of HMEC-1s. 
A
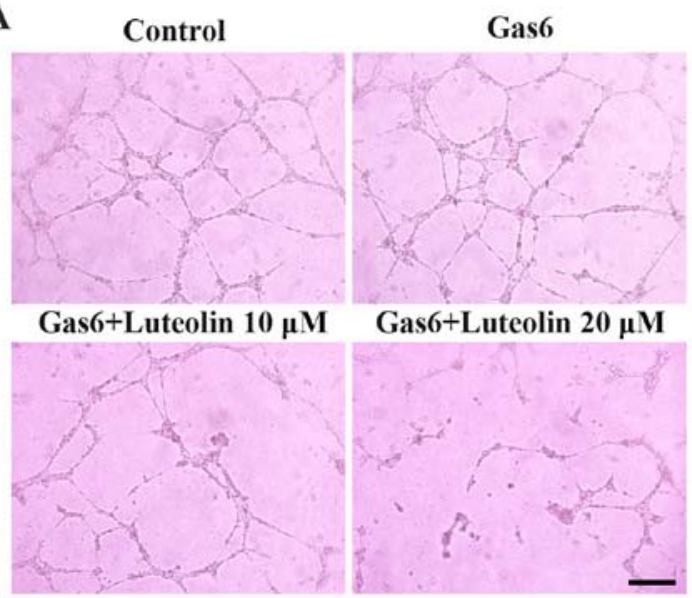

B

Control Gas6
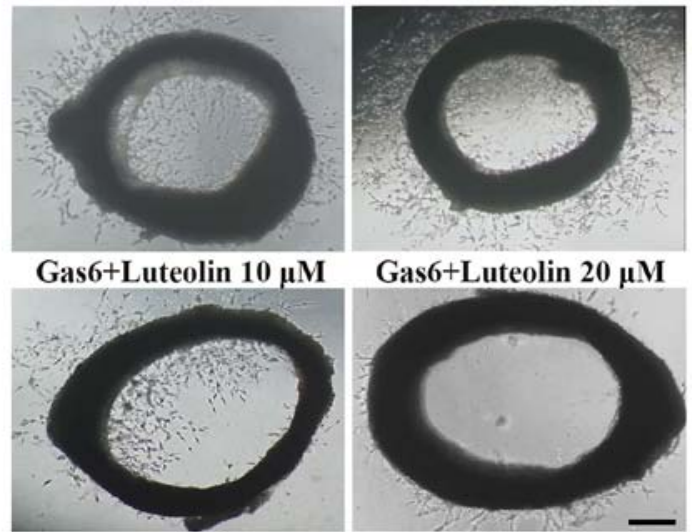

Gas6+Luteolin $20 \mu \mathrm{M}$
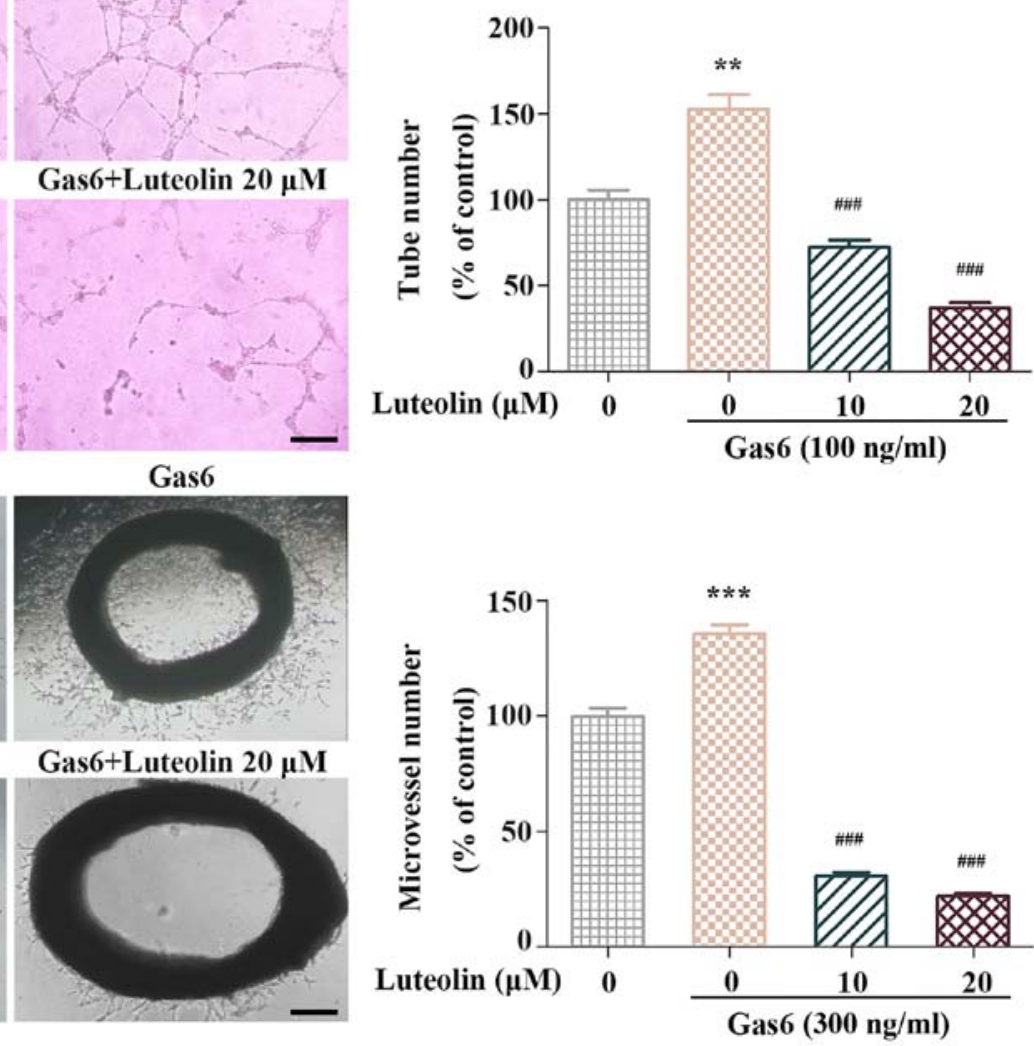

Figure 3. Luteolin suppresses Gas6-induced angiogenesis ex vivo. (A) Luteolin inhibited the Ga6-induced tube formation of the HMEC-1s. HMEC-1s (2x10 $\left.{ }^{4}\right)$ treated with the indicated concentrations of luteolin were seeded in Matrigel-coated 96-well plates. After 8-h incubation, the capillary networks were observed and photographed under an Olympus IX70 inverted microscope. The number of tubes was quantified using Image-Pro Plus 6.0. (B) Luteolin suppressed Ga6induced microvessel sprouting in the aorta ring assay. The aortic rings were treated with various concentrations of luteolin in the presence or absence of Gas6 $(300 \mathrm{ng} / \mathrm{ml})$. Representative images are shown. The microvessel outgrowth was observed and photographed under an Olympus IX70 inverted microscope, scale bar, $50 \mu \mathrm{M}$. The data are presented as the mean \pm SEM. ${ }^{* *} \mathrm{P}<0.01,{ }^{* * *} \mathrm{P}<0.001$ compared with the control group; ${ }^{\# \#} \mathrm{P}<0.001$ compared with the Gas6-treated group.

In addition, luteolin caused a concentration-dependent inhibitory effect on Gas6-induced growth of the HMEC-1s (Fig. 1B).

Luteolin inhibits the Gas6-induced migration and invasion of $H M E C-1 s$. The effect of luteolin on the Gas6-induced migration was evaluated by the wound-healing migration assay and Transwell assay. Confluent HMEC-1s were starved with serum-free RPMI-1640 medium for $6 \mathrm{~h}$ and scratched with pipette tips. Then, the cells were treated with or without luteolin and Gas6 (100 ng/ml). In the Gas6-treated group, the number of migrated HMEC-1s increased compared with the control group; however, the luteolin treatment suppressed the Gas6-induced migration of HMEC-1s (Fig. 2A). Similar results were observed in the migration chamber and invasion chamber assay. Gas6 facilitated the migration and invasion of HMEC-1s, and the stimulatory effect of Gas6 was attenuated by luteolin (Fig. 2B and C). Taken together, luteolin significantly suppressed the Gas6-induced motilities of HMEC-1s.

Luteolin inhibits the Gas6-induced angiogenesis ex vivo. We performed a tube formation assay and an aortic ring assay to examine the anti-angiogenic effects of luteolin ex vivo. In the tube formation assay, we found that the capillary-like tube networks in the Gas6-treated group were more solid and complicated than those in the control group. In contrast, luteolin significantly inhibited Gas6-induced tube formation. The number of tubes was significantly decreased after luteolin exposure (Fig. 3A). Next, we used a mouse aortic ring angiogenesis assay to investigate whether luteolin suppressed the Gas6-induced outgrowth of microvessels. The outgrowth of microvessels in the aortic ring assay was remarkably enhanced following Gas6 treatment. In contrast, luteolin markedly suppressed Gas6-induced microvessel sprouting (Fig. 3B). These results suggested that luteolin inhibited Gas6-induced angiogenesis ex vivo.

Luteolin inhibits the Gas6-induced pericyte recruitment to endothelial tubes. Since pericytes are crucial for the maturation of neovessels during late-stage angiogenesis and Axl is overexpressed in pericytes $(26,27)$, we conducted a $3 \mathrm{D}$ co-culture of HMEC-1s and HBVPs to evaluate the effect of luteolin on pericyte recruitment to the endothelial tubes. A large number of HBVPs migrated to the endothelial tubes 
A
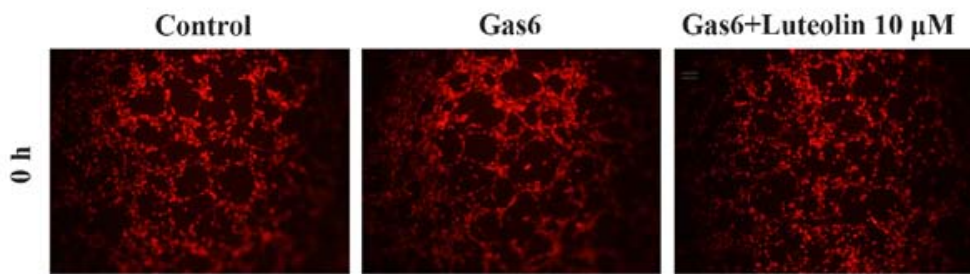

Gas6+Luteolin $20 \mu \mathrm{M}$
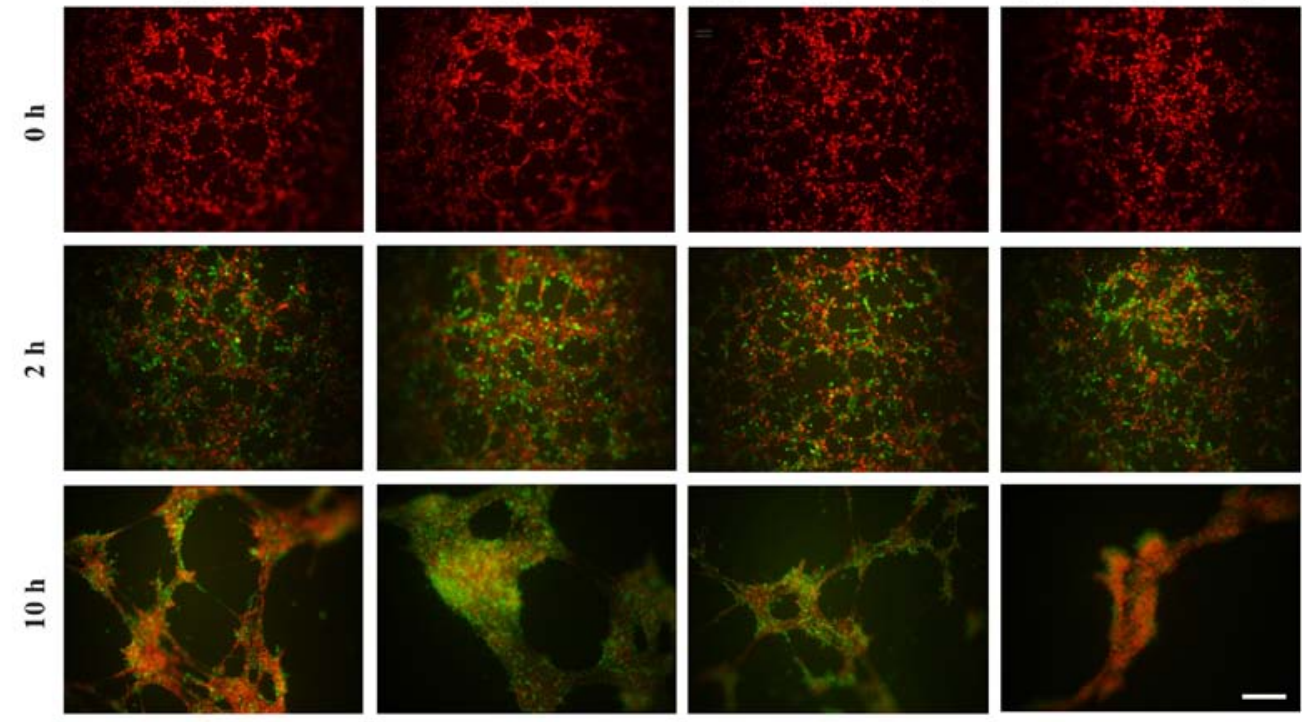

B
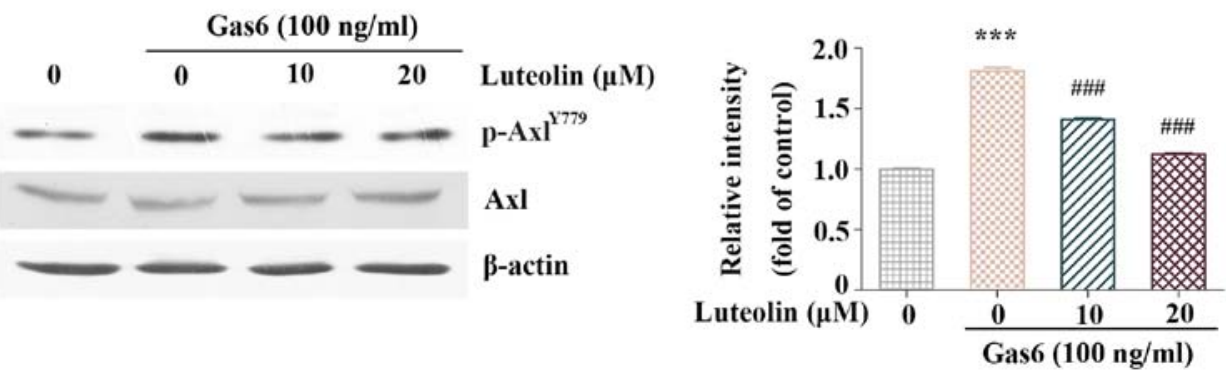

Figure 4. Luteolin suppresses Gas6-mediated HBVP recruitment to the endothelial tubes. (A) HBVPs (green) pre-treated with or without the indicated concentrations of luteolin for $4 \mathrm{~h}$, were seeded after the endothelial tubes (red) formed. The recruitment of the HBVPs to the HMEC-1 tubes was observed and photographed after the addition of HBVPs for 2 and $12 \mathrm{~h}$ in the presence or absence of Gas6. Representative images are shown, scale bar, $50 \mu \mathrm{M}$. (B) Luteolin inhibits the Gas6-induced phosphorylation of Axl in HBVPs. The HBVPs were pre-treated with luteolin for $4 \mathrm{~h}$ and then stimulated with Gas6 for another $1 \mathrm{~h}$. The quantitative analysis of western blotting was shown. Data were ratios of $\mathrm{p}$-Ax $1 / \beta$-actin and Axl/ $\beta$-actin, and were presented as mean \pm SEM. ${ }^{* * *} \mathrm{P}<0.001$ compared with the control group; ${ }^{\# \# \#} \mathrm{P}<0.001$ compared with the Gas 6 -treated group.

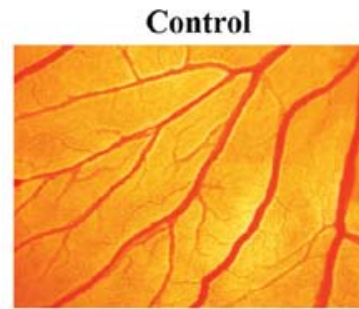

Gas6+Luteolin $10 \mu \mathrm{M}$

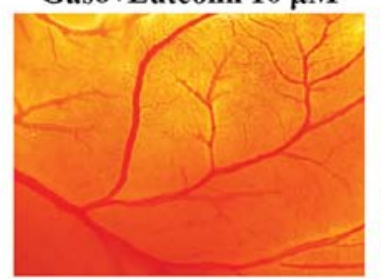

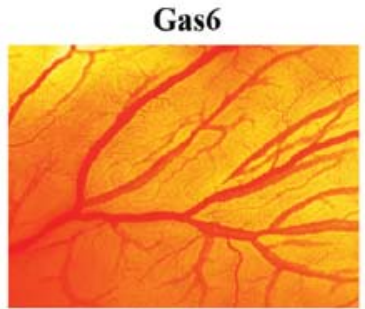
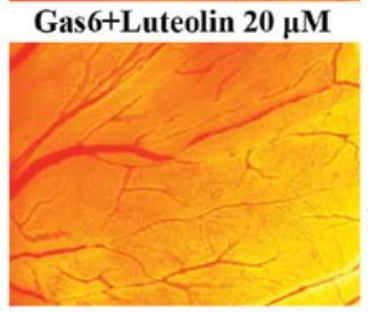

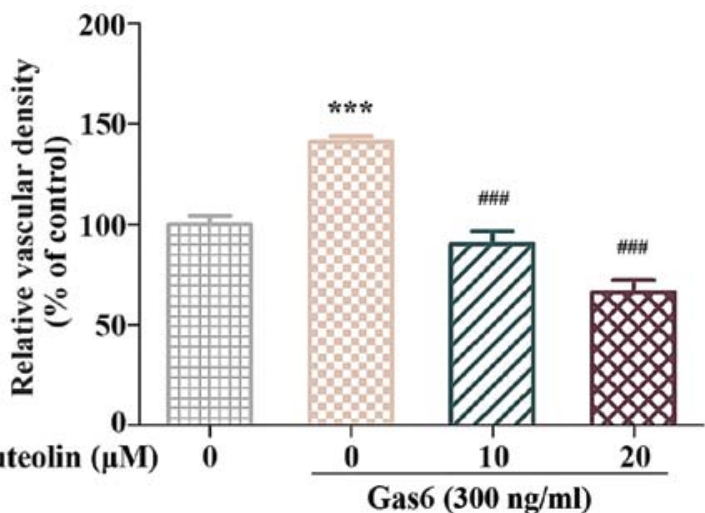

Gas6 (300 ng/ml)

Figure 5. Luteolin inhibits Gas6-induced angiogenesis in CAM. Representative images of chick chorioallantoic membrane in the CAM assay are shown. The fertilized eggs were treated with or without the indicated concentrations of luteolin in the presence or absence of Gas 6 ( $300 \mathrm{ng} / \mathrm{ml})$ for $48 \mathrm{~h}$. The microvessels were observed and captured under an Olympus SZX18 dissecting microscope (magnification, x1.6). The data are presented as the mean \pm SEM. ${ }^{* * *} \mathrm{P}<0.001$ compared with the control group; ${ }^{\# \#} \mathrm{P}<0.001$ compared with the Gas6-treated group. 
A

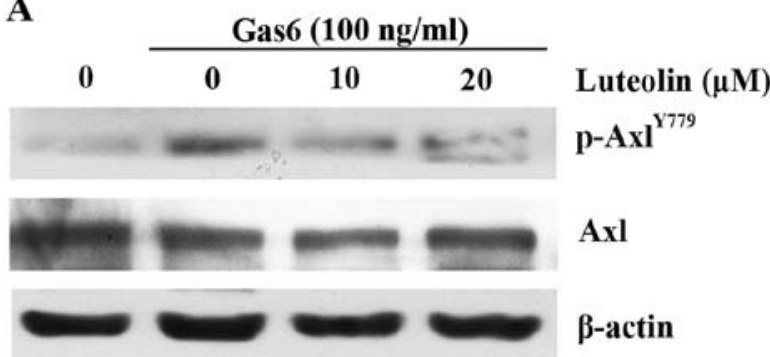

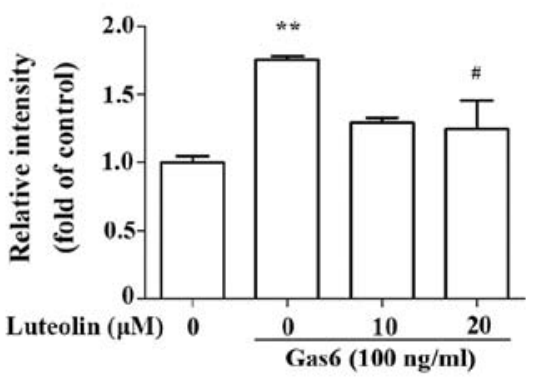

B

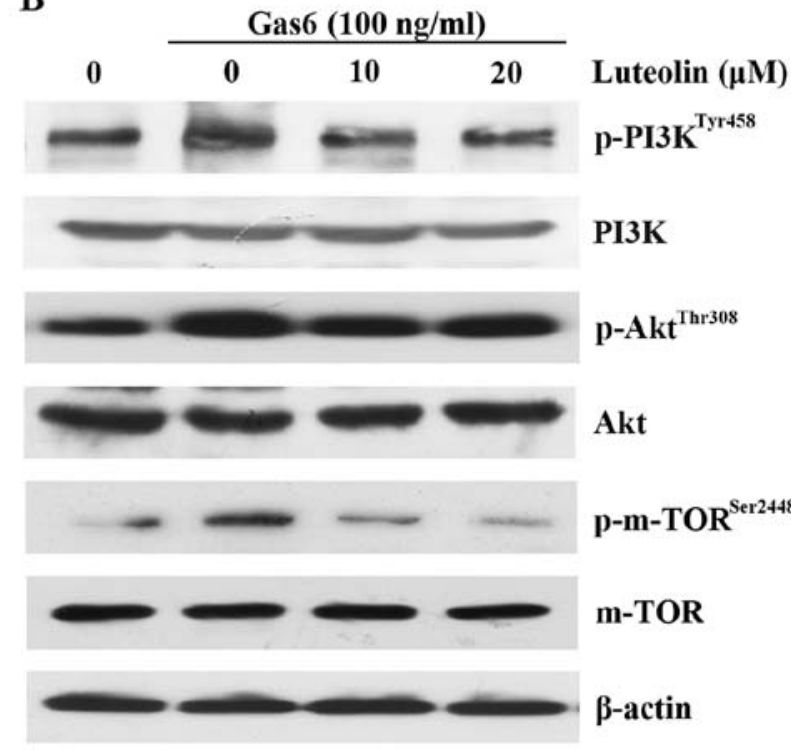

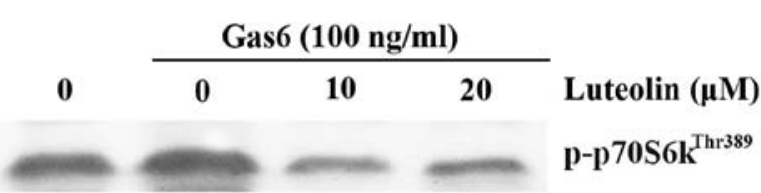
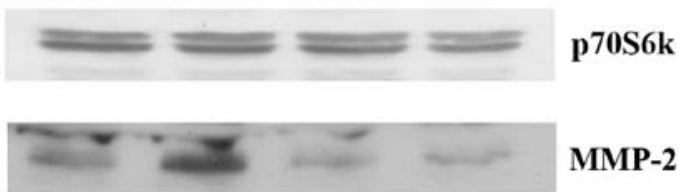

MMP-2
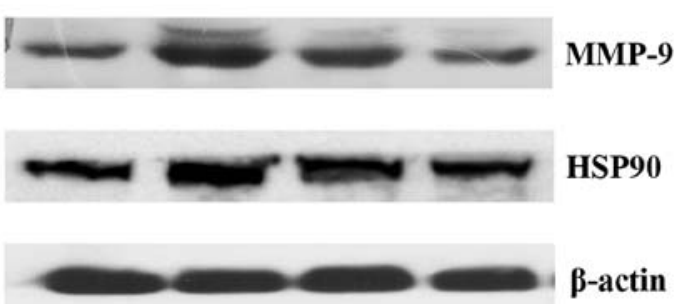

Figure 6. Luteolin suppresses the Gas6/Axl signaling pathways in HMEC-1s. HMEC-1s were pre-treated with the indicated concentrations of luteolin for 4 h and then stimulated with Gas6 $(100 \mathrm{ng} / \mathrm{ml})$ for $1 \mathrm{~h}$. Then, the cells were harvested, lysed with RIPA buffer and subjected to western blot analysis. The gray value was measured with ImageJ software. (A) Luteolin inhibited the activation of Axl in HMEC-1s. The quantitative analysis of western blotting is shown. Data were ratios of $\mathrm{p}$-Axl $/ \beta$-actin and $\mathrm{Axl} / \beta$-actin, and were presented as the mean $\pm \mathrm{SEM}$. ${ }^{* *} \mathrm{P}<0.01$ compared with the control group; ${ }^{*} \mathrm{P}<0.05$ compared with the Gas6-treated group. (B) Luteolin inhibited the Gas6-induced activation of the PI3K-Akt-mTOR pathway in HMEC-1s. Luteolin suppressed the activation of PI3K, Akt, mTOR and p70S6k and also downregulated the expression of MMP-2, MMP-9 and HS90.

within $2 \mathrm{~h}$ in the control group. Noteworthy, almost all Gas6treated HBVPs migrated and adhered to the formed endothelial tubes. However, few HBVPs were recruited to the endothelial tubes in the luteolin-treated group. In addition, the tubes in the Gas6-treated group were more complex and solid after an additional 10-h incubation compared with those in the control group. However, only a few HBVP-supported tubes were observed after the luteolin treatment. These results suggested that luteolin inhibited Gas6-induced HBVP recruitment to the endothelial tubes (Fig. 4A). Then, the inhibitory effect of luteolin on the activation of Axl in HBVPs was examined by western blotting. Our results showed that luteolin significantly suppressed the Gas6-induced phosphorylation of Axl in HBVPs (Fig. 4B).

Luteolin inhibits the Gas6-induced angiogenesis in CAM. We then conducted a CAM assay to examine the anti-angiogenic effect of luteolin in vivo. The CAM assay is a widely used and accessible system in angiogenesis studies (28). We found that the blood vessels in the Gas6-treated group formed a dense and spatially-oriented branching network, and the number of blood vessels in the embryonic neovascularization increased significantly compared with control group. However, in the luteolin-treated group, the number of blood vessels was significantly decreased compared with Gas6 group (Fig. 5). These results suggested that luteolin suppressed the Gas6-induced angiogenesis in vivo.

Luteolin inhibits the Gas6/Axl signaling pathway. The above mentioned results demonstrated that luteolin suppressed Gas6-induced angiogenesis in vitro, ex vivo and in vivo. Thus, we investigated whether this effect was associated with the suppression of the Gas6/Axl signaling pathway and explored its downstream molecular mechanisms. We found that luteolin significantly suppressed the Gas6-mediated phosphorylation of Axl, followed by the downregulation of Gas6/Axl mediated phosphorylation of the PI3K, Akt, mTOR and p70S6k (Fig. 6A and $\mathrm{B})$. Luteolin also markedly inhibited the expression of MMP-2 and MMP-9 (Fig. 6B), which play crucial roles in various physiological processes, such as wound healing and vessel sprouting (29). In addition, luteolin also inhibited the expression of HSP90 (Fig. 6B), which has been shown to 
promote angiogenesis (30). These data indicated that luteolin inhibited Gas6-induced angiogenesis by inactivating the Gas6/Axl signaling pathway in HMEC-1s.

\section{Discussion}

Epidemiological and preclinical evidence indicates that the dietary intake of flavonoids, including luteolin and catchin, contributes to the treatment of multiple pathological angiogenesis involving cancer, arthritis and cataractogenesis. Previously, luteolin has been widely investigated to validate its anti-angiogenic effects, but the underlying mechanism is not fully understood $(9,19,31,32)$. In the present study, we found that luteolin inhibited growth arrest-specific protein 6 (Gas6)-induced angiogenesis by inhibiting the Gas6/Axl signaling pathway. Noteworthy, we found that luteolin not only inhibited the Gas6-induced motilities of HMEC-1s (proliferation, migration, invasion and tubulogenesis) that are required for neovascularization but also suppressed the Gas6-induced recruitment of pericytes to well-established endothelial tubes, which is vital for the maturation of neovessels during the late-stage of angiogenesis. Our study provides new evidence regarding novel anti-angiogenic mechanism of luteolin and also contributes to the notion that the dietary intake of luteolin is beneficial for the treatment of pathological angiogenesis.

Gas6/Axl is expressed in blood vascular system containing endothelial cells (ECs), pericytes and smooth muscle cells (7-9). The Gas6/Axl pathway can promote proliferation, migration, invasion and tube formation of endothelia cells by regulating the PI3K/Akt/mTOR signaling pathway $(26,33)$. Gas6-induced angiogenesis is associated with various pathological angiogenesis, including cancer, psoriasis, blindness and arthritis $(2,34)$. Gas6 and its receptor play a crucial role in tumor angiogenesis, which functions in a variety of tumors including breast cancer, prostatic cancer and non-small cell lung cancer (3). Therefore, it is meaningful to develop an inhibitor that targets the Gas6-induced angiogenesis. Herein, we sought to determine whether the anti-angiogenic effect of luteolin is correlated with the Gas6/Axl signaling pathway. We demonstrated that luteolin significantly inhibited multiple processes of Gas6-induced HMEC-1s during angiogenesis. Luteolin also suppressed the Gas6-stimulated recruitment of HBVPs to the endothelial tubes, which promoted the maturation and stabilization of neovessels. In addition, luteolin suppressed the Gas6-induced newly branched blood vessels in the CAM. These results were due to the inactivation of the Gas6/Axl pathway, resulting in the downregulation of the $\mathrm{PI} 3 \mathrm{~K} / \mathrm{Akt} / \mathrm{mTOR}$ signaling pathway. Luteolin also reduced the expression of MMP-2, MMP-9 and HSP90, which are vital for the degradation of extracellular matrix (ECM) components (29). Taken together, our study provides new insight for further exploring the anti-angiogenic effect of luteolin and contributes to the understanding of its mechanism. Our study also indicates the potential of luteolin in the treatment of cancer, psoriasis, blindness and arthritis involvement of Gas6/ Axl activation.

Previous studies have demonstrated that the Gas6/Axl is involved in chemoresistance in breast cancer and indicated that the inactivation of the Gas6/Axl pathway may contribute to the activity of chemotherapeutic drugs $(35,36)$. Recent studies have demonstrated that the inhibition of the Gas6/Axl pathway improved the efficacy of chemotherapies in preclinical models of advanced pancreatic and ovarian cancer (37). Thus, the dietary intake of luteolin during therapy likely contributes to the decreased risk of resistance. In addition, luteolin has been shown to inhibit VEGF/VEGFR2-mediated angiogenesis $(20,38)$, combined with our results, luteolin may achieve a synergistic anti-angiogenic efficacy which is similar to that achieved by combinational therapy with inhibitors targeting the Gas6/Axl and VEGF/VEGFR2 signaling pathways in anti-angiogenesis therapies.

Currently, angiogenic inhibitors are widely used in cancer therapy in clinic, including bevacizumab, a monclone antibody of VEGF, and the multi-targeted tyrosine kinase inhibitors, such as sunitinib, and sorafenib (39). However, it was reported that many side effects emerged after the treatments, the severely adverse reactions including nausea, vomiting, and drug resistance in the advanced stage, which lead to treatment failure (40). In addition, the drugs used for the treatment of angiogenesis, such as bevacizumab and ranibizumab, are quite expensive, thus many patients can not afford the high treatment expense. However, luteolin is widely distributed in the vegetables and fruits (17), it can be obtained in our daily life as a nutritional additive thus preventing from pathological angiogenesis in clinic.

In conclusion, we demonstrate that luteolin significantly suppresses Gas6-induced angiogenesis in vitro, ex vivo and in vivo. This study investigated the potential of luteolin in clinic as a therapy for angiogenesis and it also provides evidence that dietary intake of luteolin may contribute to reducing the risk of pathological angiogenesis.

\section{Acknowledgements}

This study was supported by the Science and Technology Program of China (2012ZX09103101-053), the National Science Foundation of China (81573455) and Guangdong Province (S2013050014183 and 2013CXZDA006), the Program for New Century Excellent Talents in University and Pear River Scholar Funded Scheme (D.Z.) and the Project for Constuction of Traditional Chinese Medicine Strong Province (20132114).

\section{References}

1. Dong R, Yang GD, Luo NA and Qu YQ: HuR: A promising therapeutic target for angiogenesis. Gland Surg 3: 203-206, 2014.

2. Carmeliet P and Jain RK: Molecular mechanisms and clinical applications of angiogenesis. Nature 473: 298-307, 2011.

3. Gavalas NG, Liontos M, Trachana SP, Bagratuni T, Arapinis C, Liacos C, Dimopoulos MA and Bamias A: Angiogenesis-related pathways in the pathogenesis of ovarian cancer. Int J Mol Sci 14: 15885-15909, 2013.

4. Thanapprapasr D, Hu W, Sood AK and Coleman RL: Moving beyond VEGF for anti-angiogenesis strategies in gynecologic cancer. Curr Pharm Des 18: 2713-2719, 2012.

5. Alameddine RS, Yakan AS, Skouri H, Mukherji D, Temraz S and Shamseddine A: Cardiac and vascular toxicities of angiogenesis inhibitors: The other side of the coin. Crit Rev Oncol Hematol 96: 195-205, 2015.

6. Kariolis MS, Miao YR, Jones DS II, Kapur S, Mathews II, Giaccia AJ and Cochran JR: An engineered Axl 'decoy receptor' effectively silences the Gas6-Axl signaling axis. Nat Chem Biol 10: 977-983, 2014. 
7. Avanzi GC, Gallicchio M, BottarelF, GammaitoniL, Cavalloni G, Buonfiglio D, Bragardo M, Bellomo G, Albano E, Fantozzi R, et al: GAS6 inhibits granulocyte adhesion to endothelial cells. Blood 91: 2334-2340, 1998.

8. Manfioletti G, Brancolini C, Avanzi G and Schneider C: The protein encoded by a growth arrest-specific gene (gas6) is a new member of the vitamin K-dependent proteins related to protein $\mathrm{S}$ a negative coregulator in the blood coagulation cascade. Mol Cell Biol 13: 4976-4985, 1993.

9. Fotsis T, Pepper M, Adlercreutz H, Hase T, Montesano R and Schweigerer L: Genistein, a dietary ingested isoflavonoid, inhibits cell proliferation and in vitro angiogenesis. J Nutr 125 (Suppl 3): 790S-797S, 1995.

10. Zuo PY, Chen XL, Lei YH, Liu CY and Liu YW: Growth arrestspecific gene 6 protein promotes the proliferation and migration of endothelial progenitor cells through the PI3K/AKT signaling pathway. Int J Mol Med 34: 299-306, 2014.

11. Stenhoff J, Dahlbäck B and Hafizi S: Vitamin K-dependent Gas6 activates ERK kinase and stimulates growth of cardiac fibroblasts. Biochem Biophys Res Commun 319: 871-878, 2004.

12. Son BK, Kozaki K, Iijima K, Eto M, Nakano T, Akishita M and Ouchi Y: Gas6/Axl-PI3K/Akt pathway plays a central role in the effect of statins on inorganic phosphate-induced calcification of vascular smooth muscle cells. Eur J Pharmacol 556: 1-8, 2007.

13. Melaragno MG, Fridell YW and Berk BC: The Gas6/Axl system: A novel regulator of vascular cell function. Trends Cardiovasc Med 9: 250-253, 1999.

14. Holland SJ, Pan A, Franci C, Hu Y, Chang B, Li W, Duan M, Torneros A, Yu J, Heckrodt TJ, et al: R428, a selective smal molecule inhibitor of Axl kinase, blocks tumor spread and prolongs survival in models of metastatic breast cancer. Cancer Res 70: 1544-1554, 2010.

15. Lei X, Chen M, Nie Q, Hu J, Zhuo Z, Yiu A, Chen H, Xu N, Huang M, Ye K, et al: In vitro and in vivo antiangiogenic activity of desacetylvinblastine monohydrazide through inhibition of VEGFR2 and Axl pathways. Am J Cancer Res 6: 843-858, 2016

16. Ye X, Li Y, Stawicki S, Couto S, Eastham-Anderson J, Kallop D, Weimer R, Wu Y and Pei L: An anti-Axl monoclonal antibody attenuates xenograft tumor growth and enhances the effect of multiple anticancer therapies. Oncogene 29: 5254-5264, 2010.

17. Lin Y, Shi R, Wang X and Shen HM: Luteolin, a flavonoid with potential for cancer prevention and therapy. Curr Cancer Drug Targets 8: 634-646, 2008 .

18. Seelinger G, Merfort I and Schempp CM: Anti-oxidant, antiinflammatory and anti-allergic activities of luteolin. Planta Med 74: 1667-1677, 2008

19. Clere N, Faure S, Martinez MC and Andriantsitohaina R: Anticancer properties of flavonoids: Roles in various stages of carcinogenesis. Cardiovasc Hematol Agents Med Chem 9: 62-77, 2011.

20. Pratheeshkumar P, Son YO, Budhraja A, Wang X, Ding S, Wang L, Hitron A, Lee JC, Kim D, Divya SP, et al: Luteolin inhibits human prostate tumor growth by suppressing vascular endothelial growth factor receptor 2-mediated angiogenesis. PLoS One 7: e52279, 2012.

21. Zhang DM, Liu JS, Tang MK, Yiu A, Cao HH, Jiang L, Chan JY, Tian HY, Fung KP and Ye WC: Bufotalin from Venenum Bufonis inhibits growth of multidrug resistant HepG2 cells through G2/M cell cycle arrest and apoptosis. Eur J Pharmacol 692: 19-28, 2012

22. Ming J, Zhou Y, Du J, Fan S, Pan B, Wang Y, Fan L and Jiang J: Identification of miR-200a as a novel suppressor of connexin 43 in breast cancer cells. Biosci Rep 35: 35, 2015.

23. Darland DC and D'Amore PA: TGF beta is required for the formation of capillary-like structures in three-dimensional cocultures of 10T1/2 and endothelial cells. Angiogenesis 4: 11-20, 2001.

24. Staton CA, Stribbling SM, Tazzyman S, Hughes R, Brown NJ and Lewis CE: Current methods for assaying angiogenesis in vitro and in vivo. Int J Exp Pathol 85: 233-248, 2004.
25. Shi JM, Bai LL, Zhang DM, Yiu A, Yin ZQ, Han WL, Liu JS, Li Y, Fu DY and Ye WC: Saxifragifolin D induces the interplay between apoptosis and autophagy in breast cancer cells through ROS-dependent endoplasmic reticulum stress. Biochem Pharmacol 85: 913-926, 2013.

26. Collett G, Wood A, Alexander MY, Varnum BC, BootHandford RP, Ohanian V, Ohanian J, Fridell YW and Canfield AE: Receptor tyrosine kinase Axl modulates the osteogenic differentiation of pericytes. Circ Res 92: 1123-1129, 2003.

27. Stratman AN, Malotte KM, Mahan RD, Davis MJ and Davis GE Pericyte recruitment during vasculogenic tube assembly stimulates endothelial basement membrane matrix formation. Blood 114: 5091-5101, 2009.

28. García-Caballero M, Cañedo L, Fernández-Medarde A, Medina MA and Quesada AR: The marine fungal metabolite, AD0157, inhibits angiogenesis by targeting the Akt signaling pathway. Mar Drugs 12: 279-299, 2014.

29. Davidson B, Goldberg I, Gotlieb WH, Kopolovic J, Risberg B, Ben-Baruch G and Reich R: Coordinated expression of integrin subunits, matrix metalloproteinases (MMP), angiogenic genes and Ets transcription factors in advanced-stage ovarian carcinoma: A possible activation pathway? Cancer Metastasis Rev 22: 103-115, 2003.

30. Chang DJ, An H, Kim KS, Kim HH, Jung J, Lee JM, Kim NJ, Han YT, Yun H, Lee S, et al: Design, synthesis, and biological evaluation of novel deguelin-based heat shock protein 90 (HSP90) inhibitors targeting proliferation and angiogenesis. J Med Chem 55: 10863-10884, 2012.

31. Negrão R, Costa R, Duarte D, Gomes TT, Coelho P, Guimarães JT, Guardão L, Azevedo I and Soares R: Xanthohumol-supplemented beer modulates angiogenesis and inflammation in a skin wound healing model. Involvement of local adipocytes. J Cell Biochem 113: 100-109, 2012.

32. Sreelakshmi V, Sasikala V and Abraham A: Luteolin supplementation prevents selenite-induced cataractogenesis in Sprague Dawley rat pups. Chem Biodivers 12: 1881-1890, 2015.

33. Li Y, Ye X, Tan C, Hongo JA, Zha J, Liu J, Kallop D, Ludlam MJ and Pei L: Axl as a potential therapeutic target in cancer: Role of $\mathrm{Axl}$ in tumor growth, metastasis and angiogenesis. Oncogene 28: 3442-3455, 2009.

34. Kim YS, Jung SH, Jung DH, Choi SJ, Lee YR and Kim JS: Gas6 stimulates angiogenesis of human retinal endothelial cells and of zebrafish embryos via ERK1/2 signaling. PLoS One 9: e83901, 2014.

35. Wang C, Jin H, Wang N, Fan S, Wang Y, Zhang Y, Wei L, Tao X, $\mathrm{Gu}$ D, Zhao F, et al: Gas6/Axl axis contributes to chemoresistance and metastasis in breast cancer through Akt/GSK-3 $\beta / \beta$-catenin signaling. Theranostics 6: 1205-1219, 2016

36. Roberts CM, Tran MA, Pitruzzello MC, Wen W, Loeza J, Dellinger TH, Mor G and Glackin CA: TWIST1 drives cisplatin resistance and cell survival in an ovarian cancer model, via upregulation of GAS6, L1CAM, and Akt signalling. Sci Rep 6: 37652, 2016.

37. Lee YJ, Lim T, Han MS, Lee SH, Baek SH, Nan HY and Lee C: Anticancer effect of luteolin is mediated by downregulation of TAM receptor tyrosine kinases, but not interleukin-8, in non-small cell lung cancer cells. Oncol Rep 37: 1219-1226, 2017.

38. Bagli E, Stefaniotou M, Morbidelli L, Ziche M, Psillas K, Murphy $\mathrm{C}$ and Fotsis T: Luteolin inhibits vascular endothelial growth factor-induced angiogenesis; inhibition of endothelial cell survival and proliferation by targeting phosphatidylinositol 3'-kinase activity. Cancer Res 64: 7936-7946, 2004.

39. Yadav L, Puri N, Rastogi V, Satpute P and Sharma V: Tumour angiogenesis and angiogenic inhibitors: A review. J Clin Diagn Res 9: XE01-XE05, 2015

40. Keating GM: Bevacizumab: A review of its use in advanced cancer. Drugs 74: 1891-1925, 2014. 